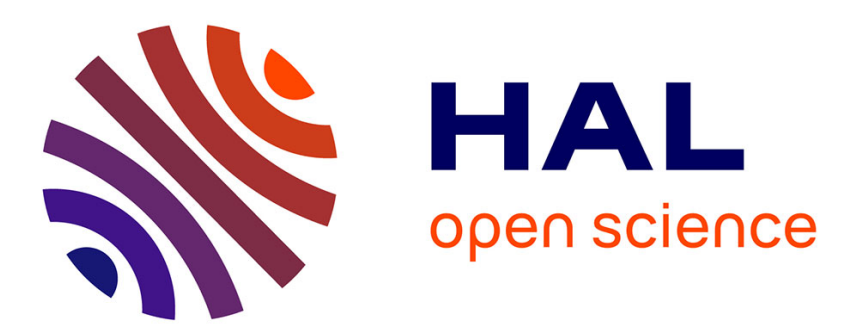

\title{
Propriétés thermométriques de couches minces de nitrure de niobium à structure désordonnée
}

Jérome Chevalier, J. Baixeras, P. Andro

\section{To cite this version:}

Jérome Chevalier, J. Baixeras, P. Andro. Propriétés thermométriques de couches minces de nitrure de niobium à structure désordonnée. Revue de Physique Appliquée, 1979, 14 (6), pp.663-667. 10.1051/rphysap:01979001406066300 . jpa-00244645

\section{HAL Id: jpa-00244645 https://hal.science/jpa-00244645}

Submitted on 1 Jan 1979

HAL is a multi-disciplinary open access archive for the deposit and dissemination of scientific research documents, whether they are published or not. The documents may come from teaching and research institutions in France or abroad, or from public or private research centers.
L'archive ouverte pluridisciplinaire $\mathbf{H A L}$, est destinée au dépôt et à la diffusion de documents scientifiques de niveau recherche, publiés ou non, émanant des établissements d'enseignement et de recherche français ou étrangers, des laboratoires publics ou privés. 


\title{
Propriétés thermométriques de couches minces de nitrure de niobium à structure désordonnée $\left({ }^{*}\right)$
}

\author{
J. Chevalier, J. Baixeras et P. Andro \\ Laboratoire de Génie Electrique des Universités Paris VI et Paris XI (**) \\ 33, avenue du Général-Leclerc, 92260 Fontenay-aux-Roses, France
}

(Reçu le 11 janvier 1979, révisé le 23 mars 1979, accepté le 23 mars 1979)

\begin{abstract}
Résumé. - Nous décrivons le comportement en fonction de la température de la conductivité de couches minces de $\mathrm{Nb}-\mathrm{N}_{x}$ élaborées par pulvérisation cathodique réactive. Les échantillons étudiés présentent les caractéristiques de matériaux désordonnés, ce qui se traduit par une variation de $\sigma(T)$ conforme à la loi de Mott concernant la conductivité par sauts thermiquement activés à distance variable entre états localisés. A partir de là nous envisageons l'intérêt potentiel de ce type de matériau pour un usage de capteur de température : la fidélité est bonne, la sensibilité excellente et peu variable en fonction de la température, le coût de fabrication serait faible ; un inconvénient pourrait venir des valeurs relativement élevées de la résistance à mesurer. En conclusion, nous pensons que ce matériau peut être très intéressant pour un usage thermométrique.
\end{abstract}

\begin{abstract}
We describe the behaviour of the conductivity of $\mathrm{Nb}-\mathrm{N}_{x}$ thin films as a function of the temperature. The samples have been prepared by reactive r.f. sputtering. The variation of $\sigma(T)$ obeys Mott's law describing the conductivity by variable range hopping between localized states in disordered materials. Then we examine the potential interest of this type of alloys in view of their use as temperature sensors : the fidelity is good, the sensitivity is excellent and varies little with temperature, the fabrication cost would be low ; the relatively high values of the resistance to be measured could be an inconvenient. In conclusion we think that this material can be very interesting for a thermometrical use.
\end{abstract}

1. Introduction. - La mesure de la température est toujours un problème délicat, qui implique généralement l'utilisation de différents types de thermomètres suivant la plage de température visée.

La valeur de la température $T$ d'un corps ne peut être obtenue qu'à partir d'une grandeur physique de ce corps dépendant elle-même de la température. Pour un thermomètre, cette variation doit être suffisamment importante (sensibilité), stable et déterminée (reproductibilité). Dans le cas des thermomètres primaires, cette grandeur physique est exprimée directement en fonction de la température thermodynamique; les thermomètres primaires les plus usités sont les thermomètres à gaz et les thermomètres à susceptibilité paramagnétique basés sur la loi de Curie. Cependant c'est la catégorie des thermomètres secondaires qui fournit la plus grande partie des thermomètres en usage. Dans ce cas, la grandeur physique

${ }^{*}$ ) Ce travail a été en partie financé par un contrat ANVARCNRS et fait partie de la thèse de doctorat de spécialité soutenue par J. Chevalier à l'Université Paris VI.

$\left({ }^{* *}\right)$ Laboratoire associé au C.N.R.S. $\mathrm{n}^{\circ} 127$. n'est pas directement liée à la température thermodynamique, et on ne peut atteindre que des variations de température. Dans cette catégorie on trouve les couples thermoélectriques, bien adaptés à la mesure de différences de température, les jonctions à semiconducteurs, et surtout les thermomètres à résistance électrique. Ces derniers se rangent en deux familles :

- les thermomètres métalliques, dont la résistance varie linéairement avec la température sur un assez large domaine,

- les thermomètres à semiconducteur, dont la résistance varie exponentiellement avec l'inverse de la température, ce qui leur confère une bonne sensibilité à basse température.

Le but de cet article est de décrire les propriétés physiques d'un alliage niobium-azote $\left(\mathrm{Nb}-\mathrm{N}_{x}\right)$, qui présente un comportement pouvant être intéressant pour la thermométrie. Le capteur de température envisagé se rangerait dans la catégorie des thermomètres secondaires à grande sensibilité.

Les composés nitrurés du niobium étudiés jusqu'à présent ont été obtenus essentiellement par pulvérisation cathodique réactive, et les travaux ont été particulièrement axés sur la phase cubique $\delta \mathrm{du} \mathrm{Nb}-\mathrm{N}$, 
qui présente un caractère supraconducteur $[1,2,3,4]$. Il a cependant été observé qu'en modifiant les conditions d'élaboration, on pouvait obtenir des couches amorphes à comportement non métallique. Ceci est très vraisemblablement lié à une augmentation sensible de la teneur en azote, qui pourrait atteindre 70 à 80 at $\%$, et qui conduit à une amorphisation du matériau. Depuis quelque temps, c'est à ce dernier type de matériau que nous nous sommes particulièrement intéressés [5] et une étude approfondie du mode de conduction dans ces couches sera présentée dans un autre article. Dans le présent article, nous allons montrer que ce type de matériau peut se comparer favorablement aux thermomètres à semiconducteur pour la mesure des températures comprises entre 4 et $300 \mathrm{~K}$.

2. Méthodes expérimentales. - La technique utilisée pour l'élaboration des couches de $\mathrm{Nb}-\mathrm{N}_{x}$ amorphe est celle de la pulvérisation cathodique réactive en montage diode r.f. La cathode est constituée de $\mathrm{Nb}$ (pureté 99,999\%) et le plasma est formé d'un mélange gazeux argon/azote à la pression de $2 \times 10^{-3}$ torr. Les paramètres de la pulvérisation, qui ont la plus grande influence sur les propriétés physiques du matériau sont la pression partielle d'azote, $\mathrm{PN}_{2}$, dans le plasma et la température, $T_{\mathrm{s}}$, du substrat, sur lequel est déposée la couche. Cette température $T_{\mathrm{s}}$ est réglée soit en chauffant le porte-substrat, soit au contraire en le refroidissant par circulation d'eau ou d'un fluide cryogénique. Les couches étudiées ont des épaisseurs de 300 à $700 \AA$ pour des temps de pulvérisation allant de $20 \mathrm{~min}$. à $1 \mathrm{~h}$. La géométrie de nos échantillons est illustrée sur la figure 1; les électrodes de contact en or sont déposées sur le substrat d'alumine préalablement à la pulvérisation.

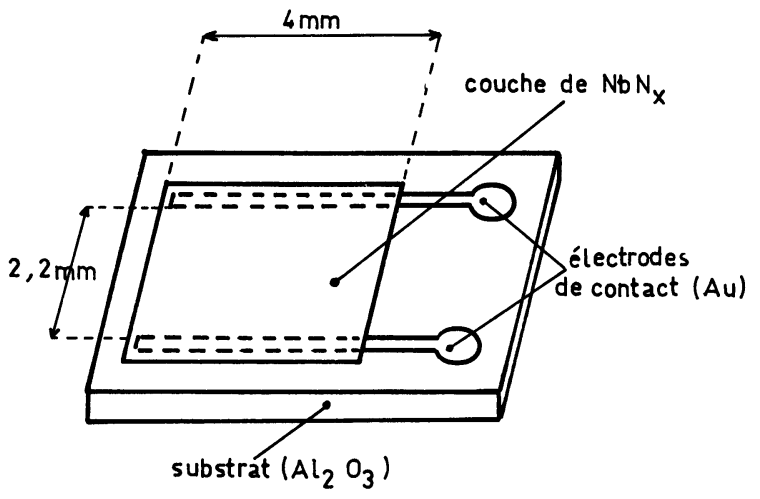

Fig. 1. - Représentation schématique de la géométrie des échantillons utilisés. L'épaisseur typique de la couche de $\mathrm{Nb}-\mathrm{N}_{x}$ est de $600 \AA$.

[Schematic view of the samples. The typical thickness of the thin film of $\mathrm{Nb}-\mathrm{N}_{x}$ is $600 \AA$.]

Les mesures de résistance en fonction de la température ont été effectuées suivant les techniques classiques de cryogénie. La valeur de la température est déterminée à l'aide d'un capteur au carbone-verre,
Lake Shore, étalonné de 1 à $300 \mathrm{~K}$; ce capteur commande un système de régulation de température. Les valeurs élevées des résistances, pouvant atteindre $10^{11} \Omega$, imposent l'utilisation d'un électromètre à grande impédance d'entrée (Keithley type 616) et nous ont conduit à vérifier que d'éventuels courants de fuite ne perturbaient pas la mesure; par ailleurs la puissance dissipée dans l'échantillon aux plus basses températures n'excède pas $10^{-10} \mathrm{~W}$.

Un point particulièrement délicat est la mesure de la magnétorésistance, car la sonde de température est elle-même magnétorésistante. Pour surmonter cette difficulté nous avons utilisé la méthode suivante : lorsque la température de l'échantillon a atteint depuis un certain temps la valeur souhaitée, nous bloquons l'intensité du courant de chauffage du porteéchantillon, le capteur de température étant déconnecté de la régulation, nous appliquons alors le champ magnétique et nous effectuons la mesure de la résistance. En pratique, pour nous assurer de la qualité des résultats ainsi obtenus, nous relevons trois valeurs successives de la résistance de l'échantillon :

$-R_{1}(0)$ résistance à champ nul, juste avant le blocage de l'intensité,

- $R(B)$ résistance en présence du champ $B$,

$-R_{2}(0)$ résistance juste après la suppression du champ B.

Si les valeurs de $R_{1}(0)$ et de $R_{2}(0)$ coïncident, nous admettons que la variation de résistance observée par suite de l'application de $B(\Delta R(B))$ n'est due qu'au champ magnétique et n'a pas été affectée par une quelconque perturbation thermique. Dans le cas où une dérive thermique ne peut être évitée au cours de l'expérience, $R_{1}(0)$ est différent de $R_{2}(0)$, dans ce cas l'incertitude relative sur la mesure de $\Delta R(B)$ peut être évaluée à $\left|R_{1}(0)-R_{2}(0)\right| /\left[\left(R_{1}(0) R_{2}(0)\right]^{1 / 2}\right.$; lorsque cette incertitude relative est inférieure à $10^{-2}$, nous admettons que la mesure est significative.

3. Résultats expérimentaux. - La première propriété intéressante à observer est la variation de la résistance électrique en fonction de la température. Le comportement typique de la plupart de nos échantillons est représenté sur la figure 2 où nous avons porté $\log R$ en fonction, soit de $T^{-1 / 4}$, soit de $T^{-1 / 2}$. On distingue généralement deux régimes de variation de la résistivité $\rho(T)$ :

— du côté des températures élevées $(T>20 \mathrm{~K})$, la résistivité obéit à la loi de Mott $[6,7]$ relative à la conduction par sauts thermiquement activés à distance variable

$$
\rho(T)=\rho_{0} \exp \left[\left(T / T_{0}\right)^{-1 / 4}\right],
$$

- pour les plus basses températures, on observe un comportement pouvant être attribué à l'existence d'un pseudo-gap de Coulomb $[8,9]$

$$
\rho(T)=\rho_{0}^{\prime} \exp \left[\left(T / T_{0}^{\prime}\right)^{-1 / 2}\right] .
$$




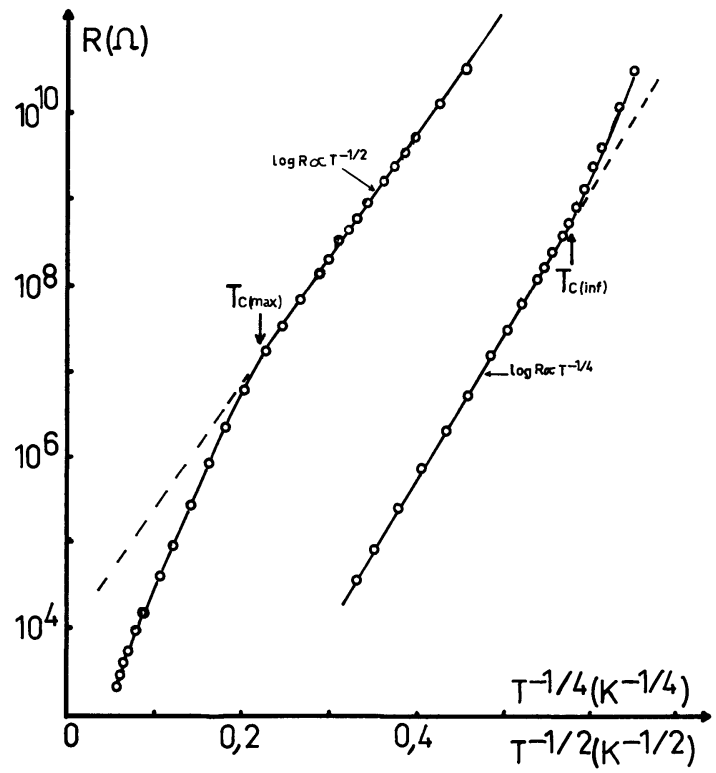

Fig. 2. - Courbes représentant la variation de la résistance d'un échantillon typique d'une part, en fonction de $T^{-1 / 4}$ (courbe de droite) et d'autre part, en fonction de $T^{-1 / 2}$ (courbe de gauche). $T_{\mathrm{c}}(\max )$ et $T_{\mathrm{c}}$ (inf) indiquent les limites expérimentales des deux comportements $R \propto \exp \left(T^{-1 / 2}\right)$ et $R \propto \exp \left(T^{-1 / 4}\right)$.

[Plots of the resistance of a typical sample versus $T^{-1 / 4}$ (right) or $T^{-1 / 2}$ (left). $T_{\mathrm{c}}$ (max) and $T_{\mathrm{c}}$ (inf) show the experimental limits of the two behaviours $R \propto \exp \left(T^{-1 / 2}\right)$ and $R \propto \exp \left(T^{-1 / 4}\right)$.]

Ces deux régimes se situent de part et d'autre d'une température critique $T_{\mathrm{c}}$ [8], qui selon les échantillons peut aller de 8 à $30 \mathrm{~K}$. Sur certains échantillons un troisième régime apparaît du côté des hautes températures ; ce régime, caractérisé par une loi de variation du type $\rho(T)=\rho_{0}^{\prime \prime} \exp \left[\left(T / T_{0}^{\prime \prime}\right)^{-1}\right]$, est séparé $d u$ régime de Mott par un large domaine de transition.

Du point de vue quantitatif, la conductivité varie de plusieurs ordres de grandeur entre $4 \mathrm{~K}$ et $300 \mathrm{~K}$. Cette dynamique est liée aux conditions d'élaboration et peut atteindre 10 ordres de grandeur.

Nous allons maintenant examiner l'aspect thermométrique des propriétés physiques de notre alliage $\mathrm{Nb}-\mathrm{N}_{x}$.

3.1 Sensibilité. - Compte tenu du comportement décrit ci-dessus, nous pouvons exprimer la résistance des échantillons sous la forme

$$
R=R_{0 x} \exp \left(b(x) T^{-1 / x}\right),
$$

où $x$ prend les valeurs 2 ou 4 selon qu'on se situe aux basses ou hautes températures. Si la sensibilité $S$ est définie par :

$$
S=\left|\frac{\mathrm{d} R / R}{\mathrm{~d} T / T}\right|,
$$

il en résulte la forme suivante de la sensibilité :

$$
S(T)=\frac{b(x)}{x} T^{-1 / x}
$$

dans les domaines correspondants de température. Typiquement, pour l'ensemble de nos échantillons on a : $20<b(x)<50$. Cette diversité de valeurs de la sensibilité est liée aux conditions d'élaboration des couches. Nous avons observé que les paramètres les plus importants sont la température du substrat et la pression partielle d'azote dans le plasma, au cours de la pulvérisation. Il est vraisemblable que la cause physique de l'influence de ces paramètres soit liée à la cinétique d'absorption de l'azote par la couche en croissance. Les deux courbes extrêmes de la sensibilité correspondant aux limites précédentes sont représentées en fonction de la température sur la figure 3, comparativement à celles de trois autres thermomètres usuels à résistance électrique [10]. La courbe supérieure du $\mathrm{Nb}-\mathrm{N}_{x}$ correspond à un échantillon obtenu à basse température de substrat et à très forte pression partielle d'azote, celle du bas en revanche correspond à un échantillon pour lequel la température du substrat est voisine de la température ambiante et la pression partielle d'azote moins élevée que la précédente. Il apparaît que la sensibilité des couches de $\mathrm{Nb}-\mathrm{N}_{x}$ amorphe d'une part est, en général, nettement plus importante, et d'autre part varie moins et de façon plus régulière que celle des autres thermomètres. Le dernier point est un avantage considérable lorsqu'on veut couvrir un large domaine de température avec un capteur unique. Si on examine la précision qu'on peut obtenir sur la mesure de la température avec l'échantillon le plus sensible en utilisant un appareil de mesure de la résistance d'une précision relative, maintenant commune, de $10^{-4}$, on arrive à

$$
\begin{array}{lll}
\frac{\Delta T}{T}=2 \times 10^{-6} & \text { à } & T=4 \mathrm{~K} \\
\frac{\Delta T}{T}=4 \times 10^{-6} & \text { à } & T=20 \mathrm{~K},
\end{array}
$$

ce qui permet d'apprécier des différences de température d'environ $10^{-5} \mathrm{~K}$ à $4 \mathrm{~K}$ et de $10^{-4} \mathrm{~K}$ à $20 \mathrm{~K}$; ces performances paraissent très satisfaisantes. Il faut

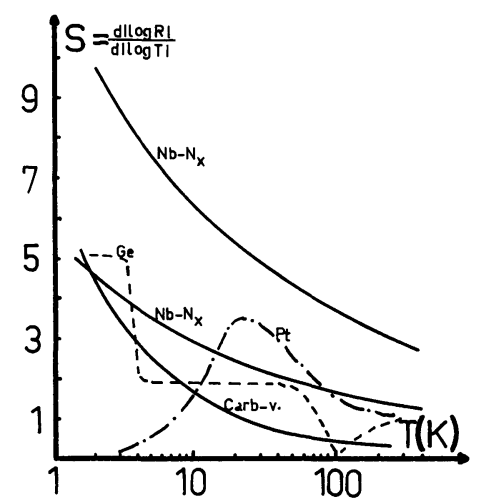

Fig. 3. - Sensibilités $s=|\mathrm{d} \log R / \mathrm{d} \log T|$ en fonction de la température pour deux échantillons de $\mathrm{Nb}-\mathrm{N}_{x}$ de comportements extrêmes en comparaison avec celles de thermomètres au germanium (Ge), au platine $(\mathrm{Pt})$ et au carbone-verre (carb. v.) [d'après R. R. Conte [10]).

[Sensitivities $s=|\mathrm{d} \log R / \mathrm{d} \log T|$ versus temperature for two samples of $\mathrm{Nb}-\mathrm{N}_{x}$ showing extreme behaviours, in comparison with the sensitivities of germanium $(\mathrm{Ge})$, platinum $(\mathrm{Pt})$ and carbonglass (carb. v.) thermometers (after R. R. Conte [10]).] 
toutefois remarquer qu'elles sont obtenues au prix de la mesure d'une résistance très élevée.

3.2 ReProductibILITÉ. - Deux types de phénomènes peuvent principalement être rendus responsables d'une mauvaise reproductibilité d'un thermomètre. D'une part, une influence du vieillissement des couches ; d'autre part, une influence des cycles thermiques impliqués par le fonctionnement même du thermomètre.

Les observations réalisées à une année d'intervalle n'indiquent aucune modification des propriétés liée au seul vieillissement des couches à la température ambiante. Par contre, les couches sont sensibles aux cyclages thermiques effectués entre la température ambiante et les basses températures. Les variations de résistance induites par le cyclage thermique dépendent de l'échantillon considéré, mais en général, la fourchette de variation n'excède pas $5 \%$ sur toute la plage de température. Les résultats reportés sur la figure 4 traduisent bien le comportement moyen de nos échantillons et il apparaît que l'évolution de la résistance des échantillons n'intéresse que les 6 à 10 premiers cyclages thermiques. Les suivants n'influent plus sur la valeur de la résistance. La reproductibilité de tels thermomètres est donc bonne à condition de prendre soin d'effectuer quelques cycles thermiques avant l'étalonnage.

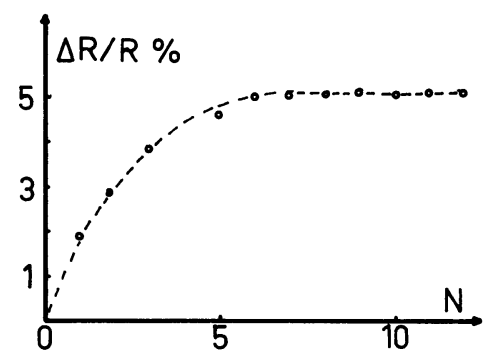

Fig. 4. - Variation de la résistance (à la température de l'azote liquide) d'une couche de $\mathrm{Nb}-\mathrm{N}_{\boldsymbol{x}}$ en fonction du nombre de cycles thermiques entre la température ambiante et $77 \mathrm{~K}$.

[Variation of the resistance (at the temperature of liquid nitrogen for a thin film of $\mathrm{Nb}-\mathrm{N}_{\boldsymbol{x}}$ as a function of the number of thermal cycles between the room temperature and $77 \mathrm{~K}$.]

Un autre problème concerne la reproductibilité des caractéristiques des couches lorsque les conditions d'élaboration restent identiques. Pour l'instant, il nous est impossible de savoir a priori quelle sera la valeur de la résistance de l'échantillon, nous ne pouvons prévoir que l'allure générale de son comportement. Il reste donc, de ce point de vue, des progrès à réaliser en vue d'une éventuelle application à la fabrication de capteurs de température.

3.3 MAGNÉTORÉSISTANCE. - La magnétorésistance des capteurs de température est un important facteur limitant leurs performances. En effet la variation de résistance due à l'existence d'un champ magnétique se traduit par une erreur sur la mesure de la tempé- rature. Il était donc important de déterminer la magnétorésistance de nos alliages $\mathrm{Nb}-\mathrm{N}_{\boldsymbol{x}}$.

Les comportements typiques de la magnétorésistance en fonction de l'intensité du champ $B$ de nos échantillons sont indiqués sur la figure 5 , aussi bien pour la magnétorésistance transversale $(B \perp J)$ que pour la magnétorésistance longitudinale $(B / / J)$. On

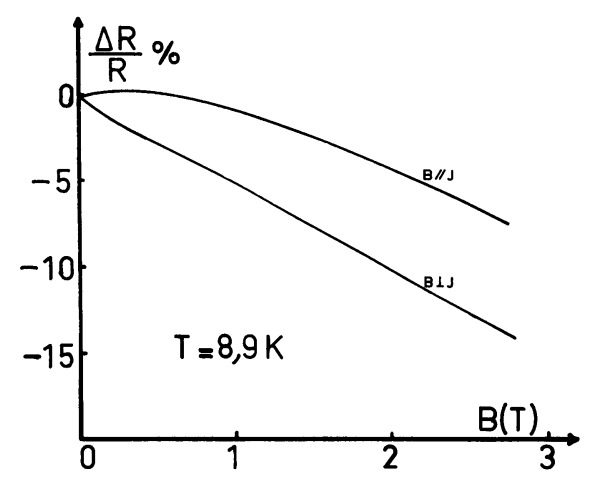

Fig. 5. - Comportement typique des magnétorésistances longitudinale et transverse en fonction du champ magnétique $B$.

[Typical behaviour of the longitudinal and transverse magnetoresistances as a function of the magnetic field $B$.]

relève que la magnétorésistance, surtout aux basses températures, est principalement négative et que sa variation en fonction du champ $B$ est approximativement linéaire aux champs forts $(B>1 T)$. On remarque en outre qu'en général, la valeur absolue de la magnétorésistance longitudinale est plus faible que celle de la magnétorésistance transversale pour les mêmes valeurs de la température et de l'intensité du champ. Par ailleurs, la magnétorésistance longitudinale est fréquemment positive, surtout aux températures élevées.

La variation, à champ constant, de cette magnétorésistance en fonction de la température (Fig. 6) est caractérisée d'une part, par l'existence d'un maximum de $|\Delta R / R|$ (situé entre 10 et $25 \mathrm{~K}$ selon les échantillons et suivant les valeurs du champ) et, d'autre part,

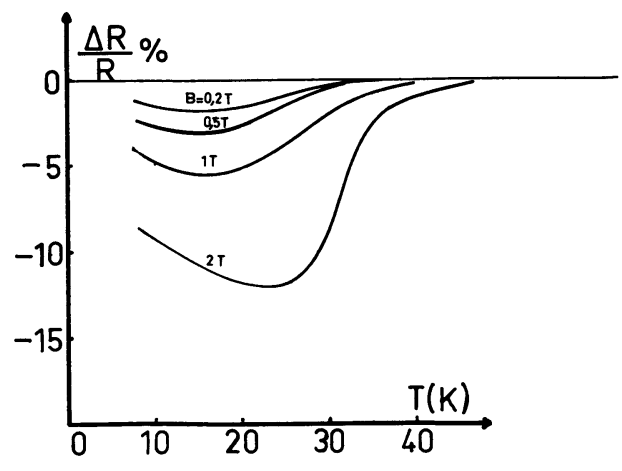

Fig. 6. - Variation de la magnétorésistance transverse en fonction de la température pour diverses valeurs du champ magnétique.

[Variation of the transverse magnetoresistance as a function of the temperature for different values of the magnetic field.] 
par une forte décroissance de cette grandeur aux températures plus élevées, la magnétorésistance devenant pratiquement nulle pour $T>40$ à $60 \mathrm{~K}$.

Pour un champ de 2,5 T, le maximum de $|\Delta R / R|$ se situe environ entre 0,1 et 0,2 pour la majorité de nos échantillons. D'un point de vue thermométrique, la magnétorésistance correspond à une erreur relative de température $|\Delta T / T|$. Nous définirons celle-ci à un champ donné égal à $2,5 \mathrm{~T}$, comme celle correspondant à $|\Delta R / R|$ maximal dans l'intervalle de champ compris entre 0 et $2,5 \mathrm{~T}$. Le plus souvent $|\Delta R / R|_{\max }$ correspond à la valeur maximale du champ (soit $2,5 \mathrm{~T}$ dans notre cas), et à la magnétorésistance transversale. Partant de la définition donnée ci-dessus de la sensibilité, il résulte :

$$
\left|\frac{\Delta T}{T}\right|=\frac{1}{S(T)}\left|\frac{\Delta R}{R}\right|_{\max } .
$$

Pour comparer les performances des couches de $\mathrm{Nb}-\mathrm{N}_{x}$ à celles des thermomètres usuels relevées par Sample et Rubin [11], nous utiliserons le $|\Delta T / T|$ correspondant à la magnétorésistance transversale maximale pour un champ magnétique $\leqslant 2,5 \mathrm{~T}$, soit

$$
\left|\frac{\Delta T}{T}\right|=\frac{1}{S(T)}\left|\frac{\Delta R}{R}\right|_{\max } .
$$

Le tableau I, sur lequel nous avons fait figurer deux échantillons de $\mathrm{Nb}-\mathrm{N}$ présentant des comportements extrêmes (cf. Fig. 3), permet d'effectuer la comparaison. On observe que les performances du Nb- $\mathrm{N}_{x}$ sont moyennes aux basses températures. En revanche, dans la plupart des cas, du fait de l'importante décroissance de $|\Delta R / R|$ aux températures plus élevées, $|\Delta T / T|$ devient très faible aux hautes températures; on obtient typiquement $|\Delta T / T| \leqslant 0,05 \%$ vers $70 \mathrm{~K}$, ce qui place le $\mathbf{N b}-\mathbf{N}_{x}$ parmi les meilleurs capteurs de température à ce point de vue.

3.4 AUtRES CRITÈreS THERMOMÉTRIQUES. - Nous avons déjà signalé l'inconvénient que représente la valeur très élevée de la résistance de nos couches de $\mathrm{Nb}-\mathrm{N}_{x}$, au niveau de la mesure. Cependant il existe une contrepartie favorable; en effet, la dissipation de puissance dans la couche nécessitée par la mesure est extrêmement faible et ne perturbe donc pas la température de l'échantillon. Par ailleurs, si on le souhaite, la résistance d'un éventuel capteur de température en $\mathrm{Nb}-\mathrm{N}_{x}$ pourrait être fortement abaissée par une configuration géométrique du type sandwich.
Tableau I. - Erreur relative de température due à la magnétorésistance pour un champ magnétique de 2,5 $\mathrm{T}$ et trois températures. Les valeurs données pour $\mathrm{Nb}-\mathrm{N}_{x}$ correspondent à deux de nos échantillons présentant des comportements extrêmes; les valeurs relatives aux autres thermomètres ont été relevées par Sample et Rubin [11].

[Relative uncertainty due to the magnetoresistance for a magnetic field of $2.5 \mathrm{~T}$ at three different temperatures. The values given for $\mathrm{Nb}-\mathrm{N}_{\boldsymbol{x}}$ correspond to two samples showing extreme behaviours, the values for other thermometers have been reported by Sample

\begin{tabular}{|c|c|c|c|}
\hline & $\begin{array}{l}|\Delta T / T| \% \\
T=10 \mathrm{~K}\end{array}$ & $\begin{array}{l}|\Delta T / T| \% \\
T=20 \mathrm{~K}\end{array}$ & $\begin{array}{c}|\Delta T / T| \% \\
T=70 \mathrm{~K}\end{array}$ \\
\hline Nature du thermomètre & $B=2,5 \mathrm{~T}$ & $B=2,5 \mathrm{~T}$ & $B=2,5 \mathrm{~T}$ \\
\hline $\begin{array}{l}\mathrm{Nb}-\mathrm{N}_{\boldsymbol{x}} \text { très } \\
\text { magnétorésistant }\end{array}$ & 2 & 3 & $\leqslant 0,05$ \\
\hline $\begin{array}{l}\mathrm{Nb}-\mathrm{N}_{x} \text { peu } \\
\text { magnétorésistant }\end{array}$ & 0,8 & 1,2 & $\leqslant 0,05$ \\
\hline Résistance au carbone & 1 & 1 & \\
\hline Carbone verre & 0,1 & 0,1 & 0,1 \\
\hline Thermistor & 0,05 & 0,05 & 0,05 \\
\hline Résistance au & 15 & 20 & \\
\hline Diode As Ga & 2 & 1 & \\
\hline
\end{tabular}
and Rubin [11].]

Un avantage d'un matériau en couche mince est sa capacité calorifique très faible; en fait la capacité calorifique du capteur serait pratiquement celle du substrat, dont on peut choisir la nature de façon à optimiser les performances du thermomètre.

4. Conclusion. - Les propriétés physiques des couches minces de $\mathrm{Nb}-\mathrm{N}_{x}$ amorphe les prédisposent à être de bons capteurs de température, dont un des principaux intérêts serait d'avoir une sensibilité élevée et variant peu en fonction de la température. Leur magnétorésistance, gênante aux plus basses températures, devient pratiquement négligeable dès que la température dépasse environ $60 \mathrm{~K}$. Notons que la simplicité et la régularité des lois de variation de $R(T)$ faciliteraient l'étalonnage d'un capteur. Enfin, le procédé d'élaboration des couches conduirait à un coût de fabrication faible.

Remerciements. - Nous remercions C. Bodin pour l'aide technique efficace qu'il nous a apportée et le Pr. G. Fournet pour ses utiles commentaires sur le manuscrit.

\section{Bibliographie}

[1] Shy, Y. M., Toth, L. E. and Somasundaram, R., J. Appl. Phys. 44 (1973).

[2] Aubert, A. et Spitz, J., Rapport C.E.N. Grenoble (1974).

[3] Wagner, W., Ast, D. and Gavaler, J. R., J. Appl. Phys. 45 (1974).

[4] Saito, Y., Anayama Moto, T. and Onodera, Y., J. Appl. Phys. 43 (1972).

[5] Baixeras, J., ANdro, P., Cazabat, M., I.E.E.E. Trans. Magn. 5 (1975) 1464.
[6] Mott, N. F., Phil. Mag. 19 (1969) 835.

[7] Motr, N. F., Phil. Mag. 24 (1970) 7.

[8] Efros, A. L. and Shklovski, B. I., Solid State Phys. 8 (1975) L-49.

[9] Baixeras, J., Chevalier, J. and Andro, P., Phil. Mag. 36 (1977) 1269.

[10] Conte, R. R., "Eléments de cryogénie ", 166 (1970).

[11] Sample, H. H. and Rubin, L. G., Cryogenics, 597 (1977). 